THE EFFECT OF DIFFUSION ON THE PHOTOCONDUCTIVTY OF THIN FILMS*

R. C. Hughes and R. J. Soke1, Sandia National Laboratories ${ }^{\dagger}$ Albuquerque, New Mexico 87185

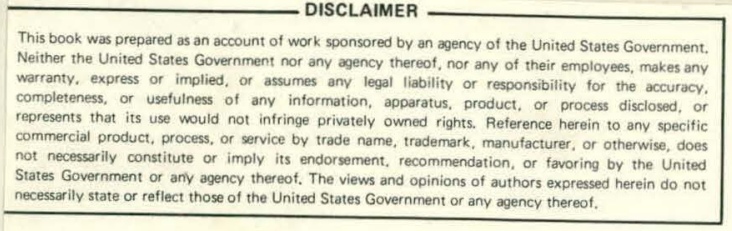

ABSTRACT

The equations governing time dependent and steady state photoconductivity are solved analytically for some approximations and numerically in exact form. The diffusion terms are shown to be important especially in thin films some data of Farmer and Lee on photoconductivity in MOS structures is reinterpreted with losses due to diffusion to the contacts included.

\title{
INTRODUCTION
}

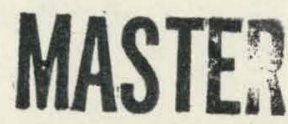

The simplest photoconductivity problem involves two metal plates, in planeparallel geometry, separated by an insulator layer with no free carriers in the dark. Electron-hole pairs are created in the insulator by band gap or other ionizing radiation and a photocurrent is observed in an external circuit when a voltage is applied. The first serious efforts to analyze the current-voltage behavior of such a device occurred in the early 20 th century with the interest in air and liquid ionization chambers.(1) Even without carrier trapping which we often face in our solid photoconductors, the complete analysis of the photoconductivity is quite complicated, and without approximations, results in differential equations which are only soluble by numerical methods. One of the first approximations that is usually made is to ignore the gradients in carrier concentrations which lead to diffusion currents in favor of using electric fields which are high enough to mask diffusion effects and also neglect variations in electric field (spatially) caused by concentration gradients in the charge carriers. A good summary of precomputer solutions and their application to ionization in liquidstes onotles has been given by Hummel and Schmidt.(1)

The approximations usually work fine for very thick insulating layers, but in thin film photoconductors and insulators, diffusion of the charge carriers to the metal (or semiconductor) contact cannot be ignored in many experimental situations. In this paper, we will discuss the results of approximate analytical solutions and exact numerical solutions to the photoconductivity equations including diffusion. We will compare the results to some published data on radiation-induced conductivity in MOS devices where diffusion was ignored in the analysis. Diffusion is also important in thicker photoconductors when trapping of one sign of carrier causes large distortions in the electric field inside of the sample.

\section{PHOTOCONDUCTIVITY EQUATIONS}

The current density in the one-dimensional case is

$$
\begin{aligned}
J & =J_{n}+J+\varepsilon \varepsilon_{0} \frac{\partial E}{\partial t}(x, t) \\
J_{n} & =e D_{n}(d n / d x)+\mu_{n} \operatorname{enE}(x) \\
J_{p} & =-e D_{p}(d p / d x)+\mu_{p} \operatorname{epE}(x)
\end{aligned}
$$

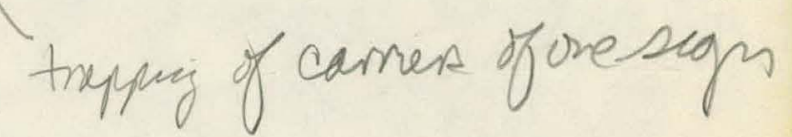

\footnotetext{
*This work was supported by the U. S. Department of Energy under Contract DE-AC04-76-DP00789.

${ }^{\dagger}$ A U. S. Department of Energy facility.
} 


\section{DISCLAIMER}

This report was prepared as an account of work sponsored by an agency of the United States Government. Neither the United States Government nor any agency Thereof, nor any of their employees, makes any warranty, express or implied, or assumes any legal liability or responsibility for the accuracy, completeness, or usefulness of any information, apparatus, product, or process disclosed, or represents that its use would not infringe privately owned rights. Reference herein to any specific commercial product, process, or service by trade name, trademark, manufacturer, or otherwise does not necessarily constitute or imply its endorsement, recommendation, or favoring by the United States Government or any agency thereof. The views and opinions of authors expressed herein do not necessarily state or reflect those of the United States Government or any agency thereof. 


\section{DISCLAIMER}

Portions of this document may be illegible in electronic image products. Images are produced from the best available original document. 
where $D_{n}$ and $D_{p}$ are the diffusion constants for electrons and holes respectively, $\mu_{n}$ and $\mu_{p}$ are the mobilities of the electrons and holes respectively (related by the Elnstein relation), $n$ and $p$ are the carrier densities for electrons and holes, and $E$ is the electric field. It is useful to note that if the system is in steady state, $J$ is a constant for all values of $x$, even though $J_{p}$ and $J_{n}$ may vary quite a bit with $x$. This relation comes from the continuity equations which govern the transient response of the system:

$$
\begin{aligned}
& \frac{\partial p}{\partial t}=D_{p} \frac{\partial^{2} p}{\partial x^{2}}-\mu_{p} \frac{\partial(E \cdot p)}{\partial x}+g-k_{p}\left(N_{t}^{O}-N_{t}^{+}\right) \\
& \frac{\partial n}{\partial t}=D_{n} \frac{\partial^{2} n}{\partial x^{2}}+\mu_{n} \frac{\partial(E \cdot n)}{\partial x}+g-\gamma n N_{t}^{+} \\
& \frac{\partial N_{t}^{+}}{\partial t}=k p\left(N_{t}^{O}-N_{t}^{+}\right)-\gamma_{t}
\end{aligned}
$$

where $\mathrm{g}$ is the rate of production of electron-hole pairs, assumed for the present to be independent of time, electric field and space; $k$ is the trapping rate of holes into a uniform distribution of neutral traps, $\mathrm{N}_{t}^{+}$; $\mathrm{N}_{\mathrm{t}}$ is the concentration of holes in those traps; and $\gamma$ is the "recombination coefficient" of electrons with those trapped holes. Other trapping or recombination rates could be included in the numerical solutions, but for the present this seems like a good approximation for insulators like $\mathrm{SiO}_{2},(2)$ and $\mathrm{PbO}$.(3) Similar approximations were made by Churchill, et al.(4) in their numerical solutions for charging of Mos capacitors. The correct solutions to these differential equations requires that Poisson's equation be satisfied

$\nabla^{2} V(\dot{x})=\frac{-e}{\varepsilon \varepsilon_{0}}\left(p-n+N_{t}^{+}\right)=\frac{-e}{\varepsilon \varepsilon_{0}} \rho(x)$

where $V$ is the potential (including any applied bias) and $\varepsilon$ is the dielectric constant for the insulator and $\varepsilon_{0}$ is the permitivity of free space.

Some of the approximate solutions are given in Ref. 1, including the simple high field Ilmit, $J=$ geL, with $L$ the thickness of the insulator. Another important specification is the boundary conditions at $x=0$ and $x=L$, where the carriers are extracted at the electrodes. The simplest boundary condition is to let $\mathrm{n}(0, \mathrm{~L})=\mathrm{p}(0, \mathrm{~L})=0$ which has been used in most plasma physics and ionization chamber problems. The rationale is that the electrons, holes or ions are unstable energetically with respect to the metal or semiconductor contact, so the recombination velocity should be high. It is possible to use a recombination rate constant (often called the surface recombination velocity in semiconductor problems), but all values of the rate constant above a certain level give the same results as the simple condition and without more specific knowledge of the rate constant it does not seem necessary to introduce a more complicated boundary condition.

A solution of interest which has not appeared anywhere to our knowledge is the one in which the steady state space charge is not sufficient to disrupt the initial field, $E=V / L$ where $V$ is the applied bias. Also letting $k, \gamma=0$ and assuming steady state, the current-voltage characteristic is

$\frac{\mathrm{J}}{\mathrm{geL}}=\frac{\mathrm{e}^{\mathrm{V} / \mathrm{kI}}+1}{\mathrm{e}^{\mathrm{V} / \mathrm{kT}}-1}-\frac{2 \mathrm{kT}}{\mathrm{V}}$

given as the ratio of the current to the saturation current. It should be noted that the solution is independent of the magnitudes of the diffusion constants, $D_{n}$ 
and $D_{p}$. Of course, if the difference in $D^{\prime} s$ leads to a significant space charge distortion of $E$, the solution will no longer be valid. However, for thin films, extraordinarily high values of $g$ have to be used to generate sufficiently high concentrations of carriers in thin films to cause a deviation from Eq. 8. For example, in a $5000 \mathrm{~A} \mathrm{SiO}_{2}$ film with $\mathrm{D}_{\mathrm{n}}=.5 \mathrm{~cm}^{2} / \mathrm{s}$ and $\mathrm{D}_{\mathrm{p}}=10^{-7} \mathrm{~cm}^{2} / \mathrm{s}$ and $\mathrm{g}=10^{14}$ and $\mathrm{V}=.05$ volt, the numerical solution with no trapping is quite close to the prediction of Eq. 8 .

\section{NUMERICAL SOLUTION}

If transient or pre-steady state solutions are desired [or steady state with appreciable space charge distortion of $E(x)$ ], then numerical analysis is required. Specifically, the particle density and current density for both electrons and holes, the trapped charge density, and the electric field as functions of position and time constitutes a complete solution to Eqs. 4 through 6 . In addition, the total current, which is the sum of the particle currents and the displacement current (which is only a function of time) is calculated. The current voltage characteristic is derived from the value of the total current in steady state.

A standard procedure for solving partial differential equations numerically is followed. The continuity equations are discretized with respect to the spatial variable according to a scheme introduced to model transport in a $\mathrm{p}$-n junction. (5) This scheme avoids potential unphysical results which can occur if conventional linear difference approximations are used. Spatial discretization of the continuity equations reduces the partial differential equations into a set of first order ordinary differential equations in the time variable which can then be easily solved with techniques for solving initial value problems. Because the electron mobility and the hole mobility can differ by many orders of magnitude, different time constants are introduced and the systemn is said to be "stiff." Thus, special care must be taken to obtain accurate transient solutions. A differential equation solver based on the work of Gear has been employed for this purpose.(6)

Several approaches for solving Poisson's equation numerically are available. The method here takes advantage of the fact that in one dimension Poisson's equation can be integrated analytically to obtain $\mathrm{E}$ in terms of the charge density $\rho$,

$E(x)=\frac{1}{\varepsilon \varepsilon_{0}} \int_{0}^{x} \rho\left(x^{\prime}\right) d^{\prime}-V / L-\frac{1}{\varepsilon \varepsilon_{0}} \int_{0}^{L}\left(1-x^{\prime} / L\right) \rho\left(x^{\prime}\right) d x^{\prime}$

where $\mathrm{V}$ is the applied voltage. This result is used to eliminate $\mathrm{E}$ in favor of $\mathrm{p}$ and $\mathrm{n}$ in the continuity equations.

A simple expression for the total curent, J, is obtained by differentiating Eq. (9) with respect to $t$ and comparing the result to the definition of the total current. It is found that

$J=L^{-1} \int_{0}^{L}\left(J_{p}+J_{n}\right) d x+\frac{\varepsilon \varepsilon_{0} d}{d t}(V / L)$

The total current is the spatial average of the particle currents plus the displacement current due to a time varying external field. This result follows from the fact that the total current is a function of $t$ only. It can be extended to three dimensions if the integral is understood to be replaced by a path integral. 
Thin $\mathrm{SiO}_{2}$ films provide a difficult test of the numerical solutions since the electron and hole mobilities are known from transient experiments to be orders of magnitude different. Our code has had no trouble giving the correct predictions for cases which can be solved analytically like Eq. 8, despite the "stiffness" of the difference equations thus giving us confidence in the time dependent and nonlinear regions. A few years ago Farmer and Lee(7) published data for steady-state photocurrents in MOS devices and had problemsjinterpreting the data at low-electric field strengths. Their data, normalized to gel or relative yield, is given in Fig. 1 for two oxides of thickness 1250 and 5000 A. They did not take diffusion Into account, and their only loss mechanisms were geminate recombination and trapping. The dashed lines in Fig. 1 contain the effect of diffusion and the known geminate recombination yield (which is field dependent). (2) Note that the thickness dependence appears in this method of plotting yields because the abscissa is in field rather than voltage units. Even the most perfect oxide, without any traps, would be expected to have a relative yield no larger than the dashed lines, unless " $g$ " was so large that bulk recombination between free electrons and holes could occur. The $\mathrm{g}$ in the Farmer and Lee experiments is about $10^{14} \mathrm{~cm}^{-3} \mathrm{~s}^{-1}$, and it would require a $\mathrm{g}$ of about $10^{18}$ to bring the steady state concentrations up to the point where bulk recombination would be important.

The dashed curve is a pretty good fit to the thinner (1250 A) oxide, which indicates that trapping is not too important. However, the fact that the thicker oxide yield falls far below the diffusion curve indicates that some trapping needs to be included in the model. The solid line gives the numerical solutions for the yield for the following paramęters: hole trapping $-\mathrm{k}=10^{4} \mathrm{~s}^{-1}$, hole trap density $-10^{18} \mathrm{~cm}^{-3}, C=10^{-7} \mathrm{~cm}^{3} / \mathrm{s}, D_{\mathrm{n}}=.5$ and $D_{\mathrm{p}}=10^{-7} \mathrm{~cm}^{2} / \mathrm{s}$. The data in Fig. 1 was taken from the integrated charge during a $50 \mathrm{~ms}$ exposure to $x$-rays and is thus only steady state in the sense that $50 \mathrm{~ms}$ is long enough for the sweep out and diffusion of the free carriers to come to steady state. The numerical solutions show that $50 \mathrm{~ms}$ is long enough if one ignores the dispersive transport of the holes, which our previous data shows is not too bad an approximation. The trapped hole concentration is of course not in steady state but is building up a constant rate for all the fields and thickneses shown in Fig. 1. At a time later than $50 \mathrm{~ms}$ the trapped holes cause a severe distortion in the electric field and at a much later time a real steady state is reached. The numerical solutions for the time dependence of the total current are given in Fig. 2 for the thicker oxide, showing the transition from the early "steady" state to the real steady state. The trapping brings the two thicknesses much closer together in relative yield, emphasizing the fact that trapping and diffusion have the opposite effect on yield vs. thickness. An increase in trapping rate for holes does not bring the $5000 \mathrm{~A}$ curve any lower in the low-field regime and some electron trapping must be added to make the curve coincide with the data. An electron trapping rate of about $4 \times 10^{19} \mathrm{~s}^{-1}$ is needed. There is no other evidence for a short electron lifetime in these oxides, although the fact that the thicker oxide was produced by a different company than the thin oxide make it plausible that the electron- and hole-lifetimes are different in the two kinds of oxide.

The decay in current as steady state is approached has been seen in a number of films including $\mathrm{SiO}_{2}$, (7) $\mathrm{PET}$, polystyrene and other polymer films, (8) but data has not been taken over sufficient time to accurately check the nodel (note the log time abscissa). The reported current decays have the general shape predicted in Fig. 2, and we plan future studies to see if consistency can be found between transient transport parameters and the long term steady state current. Other areas where the full photoconductivity code will be helpful include predicting flatband shifts in field effect devices, photocurrents and efficiencies in thin film solar cells, thermally stimulated currents in insulating films and behavior 
of electrets. There are additional physical effects which will be incorporated into the code. These include: (a) geminate recombination which implies that the value of $g$ will change in different parts of the sample as the space charge distorts the electric field; (b) field induced injection from the metal-insulator contact - a new source of carriers in the problem; (c) the dispersive transport of carriers which is common in amorphous materials; and (d) non-uniform trap distributions.

In summary, we have shown that diffusion can have important effects on the photocondutivity of thin films and that numerical solutions to the photoconductivity equations can give new insight into complicated behavior of MIM and MIS structures under excltation.

\section{REFERENCES}

1. A. Hummel and W. F. Schmidt, Radiation Research Review 5, 199 (1974).

2. R. C. Hughes, Solid State Electronics 21, 251 (1978).

3. A. M. Goodman and A. Rose, J. Appl. Phys. 42, 2823 (1971).

4. J. N. Church111, F. E. Holmstrom and T. W. Collins, J. App1. Phys. 50, 3994 (1979).

5. D. L. Scharfetter and H. K. Gumme1, IEEE Trans. Electron. Devices 16,64 (1969).

6. A. C. Hindmarsh, Computer Code GEAR (Lawrence Livermore Lab, Livermore, CA, 1974).

7. J. W. Farmer and R. S. Lee, J. Appl. Phys. 46, 2710 (1975).

8. H. Maeda, M. Kurashige and T. Nakakita, J. App1. Phys. 50, 7247 (1979).

\section{FIGURE CAPTIONS}

Fig. 1. The relative photoconductivity yield for a $1250 \mathrm{~A}$ oxide ( $)$ and a 5000 A oxide $(O)$ vs. applied field. The dashed lines are the theory predictions for geminate reconbination and diffusion alone, and the solid lines are the numerical solutions for trapping with parameters given in the text.

Fig. 2. The numerical solutions for the time dependence of the photocurrent for the 5000 A oxide for the given fields. Parameters are given in the text. 


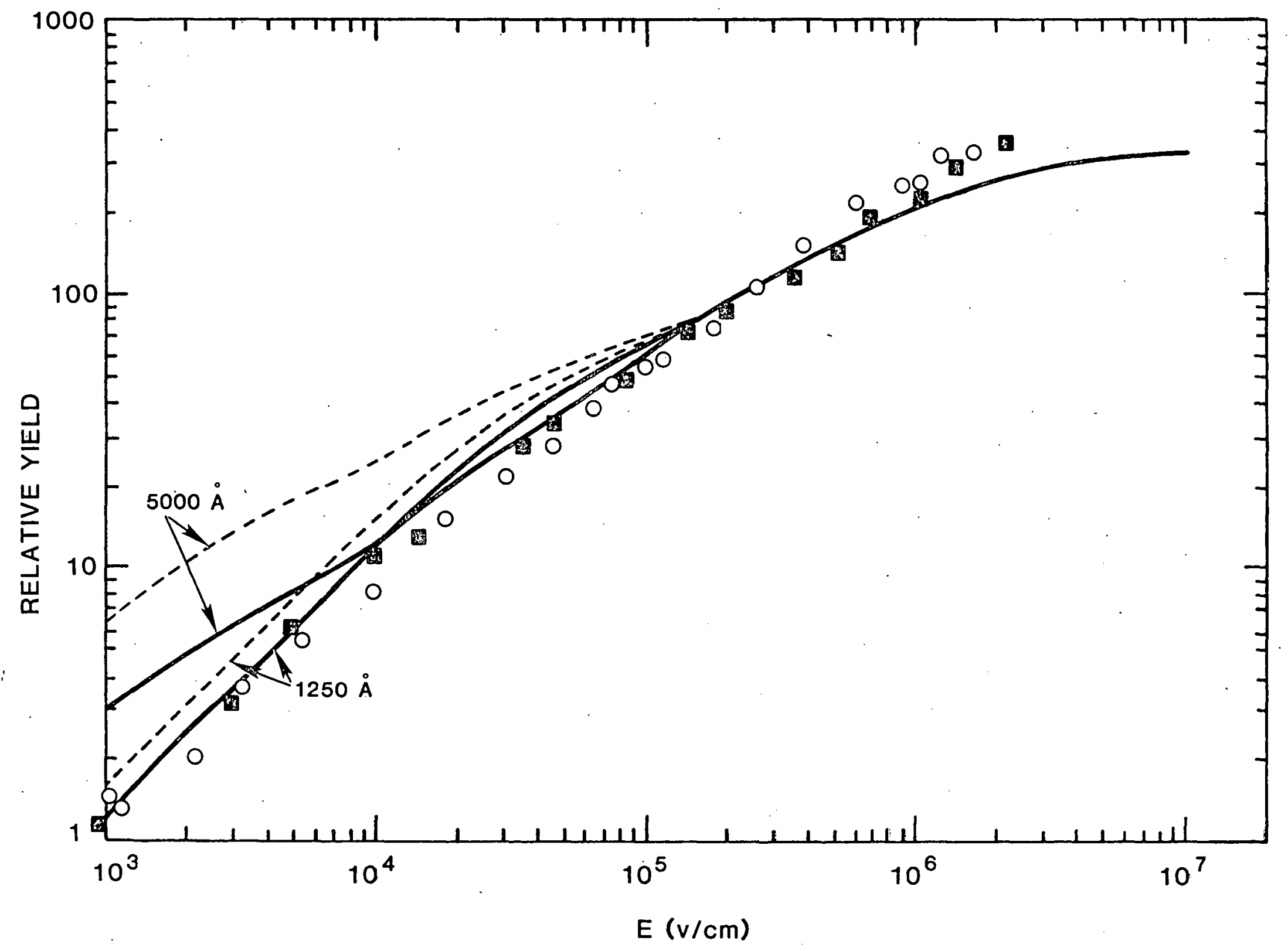




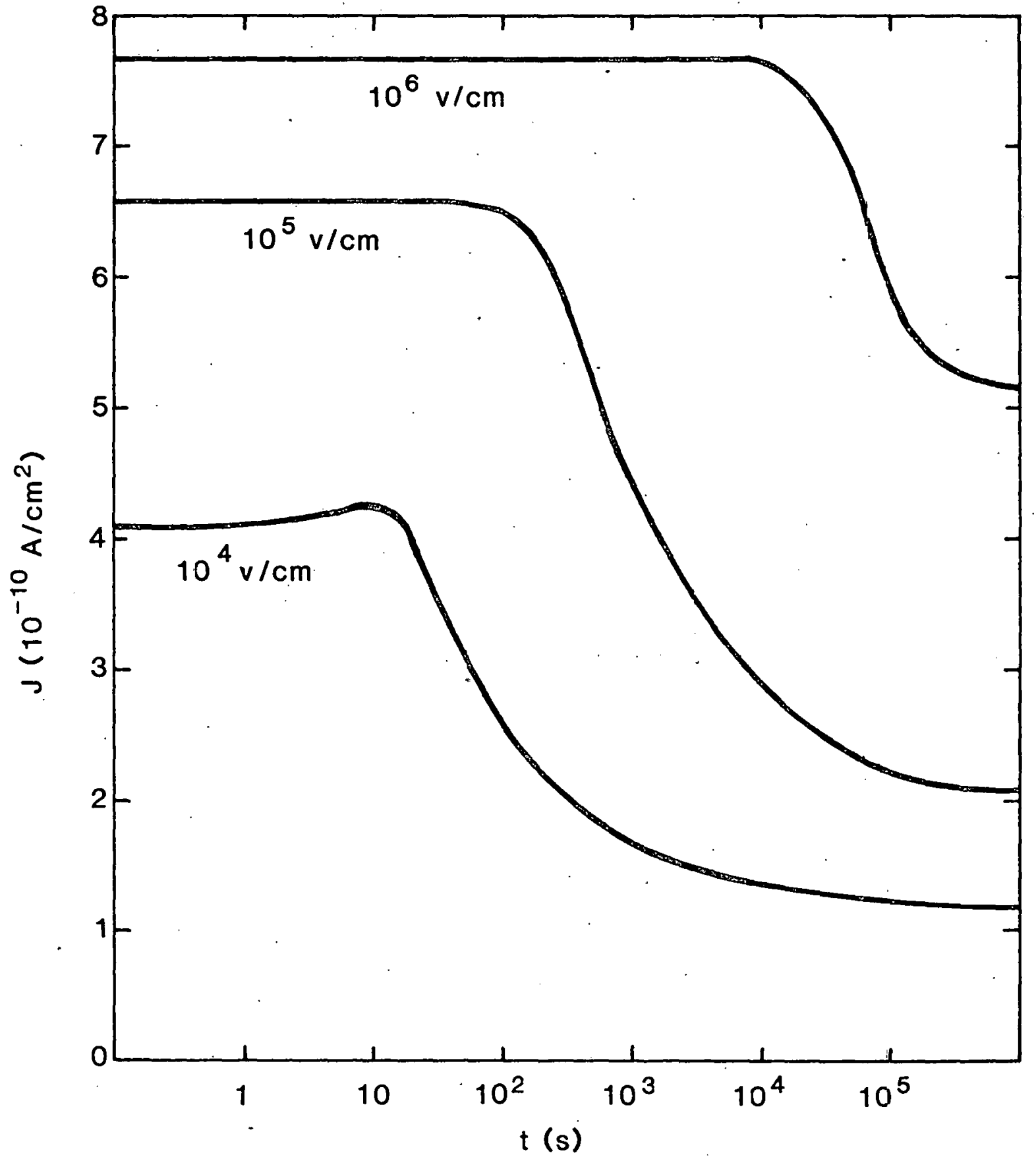

